\title{
EEG Biomarkers of reduced inhibition in human cortical microcircuits in depression
}

\author{
Frank Mazza $^{1,2}$, Taufik A. Valiante ${ }^{3,4,5,6,7,8}$, John Griffiths ${ }^{1,9,10}$, Etay Hay ${ }^{1,2,9}$ \\ ${ }^{1}$ Krembil Centre for Neuroinformatics, Centre for Addiction and Mental Health, ${ }^{2}$ Department of Physiology, University of Toronto, ${ }^{3}$ Krembil \\ Brain Institute, University Healthy Network, ${ }^{4}$ Department of Electrical and Computer Engineering, University of Toronto, ${ }^{5}$ Institute of \\ Biomaterials and Biomedical Engineering, University of Toronto, ${ }^{6}$ Department of Surgery, University of Toronto, ${ }^{7}$ Center for Advancing \\ Neurotechnological Innovation to Application, ${ }^{8}$ Max Planck-University of Toronto Center for Neural Science and Technology, ${ }^{9}$ Department of \\ Psychiatry, University of Toronto, ${ }^{10}$ Institute of Medical Sciences, University of Toronto
}

\begin{abstract}
Reduced cortical inhibition by somatostatin-expressing (SST) interneurons has been strongly associated with treatment-resistant depression. However, whether the effects of reduced SST inhibition on microcircuit activity have signatures detectible in electroencephalography (EEG) signals remains unknown. We simulated resting-state activity and EEG using detailed models of human cortical layer $2 / 3$ microcircuits with normal (healthy) or reduced SST inhibition (depression). Healthy microcircuit models showed emergent key features of resting-state EEG, and depression microcircuits exhibited increased theta, alpha and low beta power $(4-15 \mathrm{~Hz})$. The changes in depression involved a combination of an aperiodic broadband, and periodic theta and low beta components. In both conditions, neuronal spiking showed a spike preference for the phase preceding the EEG trough. Our study thus links SST inhibition level to features in EEG simulated from detailed human microcircuits, which can serve to better identify mechanistic subtypes of depression using EEG, and non-invasively monitor modulation of cortical inhibition.
\end{abstract}

Corresponding author: Dr. Etay Hay

Krembil Centre for Neuroinformatics, Centre for Addiction and Mental Health 250 College St, Toronto, Ontario, M5T 1R8

Email: etay.hay@camh.ca 


\section{Introduction}

Major depressive disorder (depression) is a leading cause of disability worldwide[1] and involves varying mechanisms and symptoms[2,3]. Consequently, a significant proportion of patients remain resistant to antidepressants[4] and second-line treatments[5]. Electroencephalography (EEG) offers a non-invasive and cost-effective method for brain signal-based biomarkers to improve diagnosis, monitoring and treatment of depression subtypes[6,7]. However, the multi-scale mechanisms of depression and their link to clinically-relevant brain signals remain poorly understood.

There is growing evidence that reduced cortical inhibition plays a critical role in treatmentresistant depression, especially inhibition mediated by somatostatin-expressing (SST) interneurons[8-13]. Recent studies showed a marked reduction in SST expression by SST interneurons in post-mortem tissue of depression patients, across all layers of the prefrontal cortex (PFC), indicating a decrease in SST inhibition[13-16]. In rodent models, silencing SST-mediated inhibition led to depression symptoms[12], and novel therapeutic compounds acting via positive allosteric modulation of alpha-5-gamma-aminobutyric-acid-A $\left(\alpha 5-\mathrm{GABA}_{\mathrm{A}}\right)$ receptors targeted by SST interneurons resulted in pro-cognitive and anxiolytic effects[12,17]. Changes in SSTmediated inhibition on circuit activity could have signatures detectible in EEG, due to this cell type's principal role in modulating input to pyramidal (Pyr) neurons, which are the primary contributors to EEG signals[8,18]. SST interneurons provide synaptic and tonic inhibition onto the apical dendrites of Pyr neurons[19,20], and mediate lateral inhibition in the cortex[8,21,22], particularly during periods of quiet, resting wakefulness (resting state) [23]. Accordingly, previous studies indicate that reduced SST inhibition in depression increases baseline activity of Pyr neurons[9,24].

While the contribution of SST interneurons to resting-state cortical oscillations remains largely unknown, studies show a role for this cell type in modulating low-frequency oscillations. SST stimulation entrains network activity in the 5 - $30 \mathrm{~Hz}$ range, and SST suppression modulates theta band $(4-8 \mathrm{~Hz})$ power[25,26]. SST interneurons have also been suggested to govern network synchrony in slow-wave sleep, which is marked by slow oscillations[27]. Thus, reduced SST inhibition in depression may affect EEG low-frequency power[28]. We recently showed that a $40 \%$ reduction in SST inhibition, estimated from post-mortem tissue studies of depression 
patients[13], significantly increased baseline Pyr spike rate in simulated human microcircuits[24]. However, it is still unknown if this level of reduction in SST inhibition would significantly alter baseline oscillatory dynamics detectable in EEG.

Previous studies have linked neuronal spiking to extracellular signals, although mostly using animal models and local field potential (LFP)[29-32]. Human studies have characterized LFP oscillations in cortical slices and showed phase-amplitude coupling between deep and superficial layers[33]. Others have studied the phase preference of Pyr neuron spikes relative to spindle events in intracranial EEG oscillations[34,35]. However, experimental methods are limited in their ability to characterize the effects of the cellular changes in depression on human brain signals in vivo, thus meriting the use of computational models. The increased availability of human neuronal electrical and synaptic connectivity data[22,36,37], provides important constraints on detailed models of human cortical microcircuitry[24] which can be used to simulate microcircuit activity in health and disease, including local circuit-generated EEG signals[38-40].

In this study, we identified EEG biomarkers of microcircuit effects due to reduced SST inhibition, as estimated from gene expression changes in depression. Using biophysically detailed models of human cortical L2/3 microcircuits, we simulated resting-state activity in health and depression together with local EEG signals. We characterized changes in resting-state EEG spectral power, and in relation to spiking activity in different neuron types, to identify biomarkers of reduced SST-mediated inhibition in depression.

\section{Results}

\section{Human L2/3 microcircuit models reproduce human EEG features}

We used our previous detailed models of human cortical L2/3 microcircuits[24] (Fig 1a) to simulate resting state spiking and EEG signals. The model microcircuits included the four key neuron types: Pyr neurons, SST interneurons, Parvalbumin-expressing interneurons (PV), and Vasoactive intestinal polypeptide-expressing interneurons (VIP). To simulate intrinsic healthy resting-state spiking activity, all neurons received random background excitatory input corresponding to baseline cortical and thalamic drive. The model microcircuits were implemented in a physical volume, enabling simulation of LFP and EEG together with microcircuit spiking (Fig 1a-d). 
A
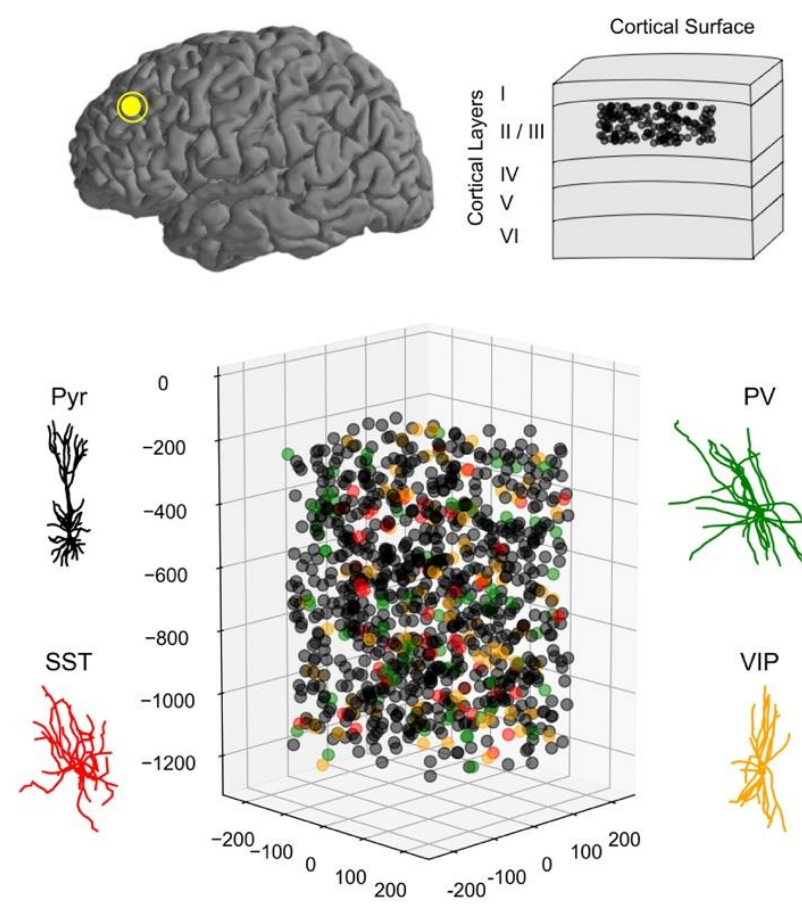

B

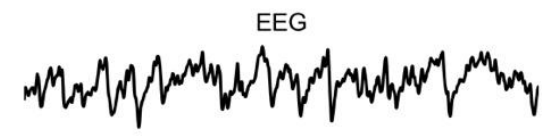

C

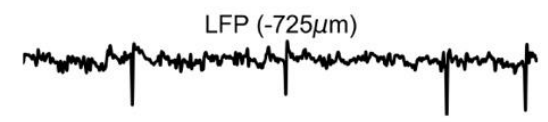

D
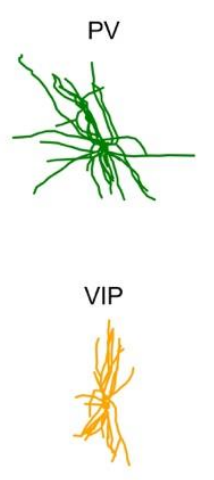

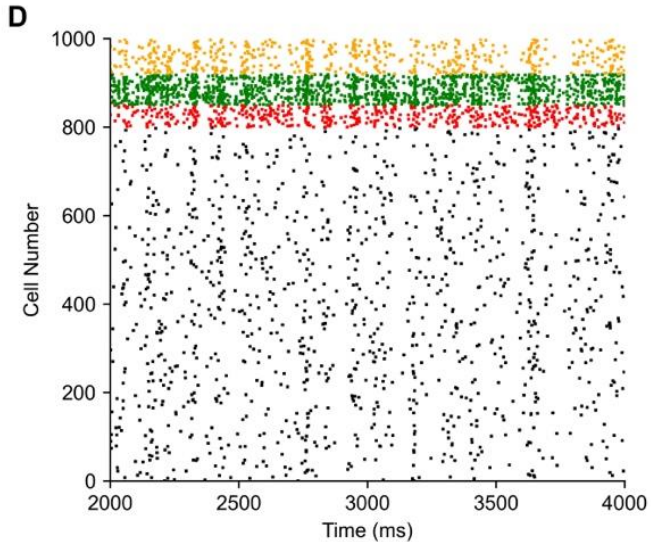

Figure 1. Simulating neuronal spiking and EEG signals from human cortical microcircuits. (a) Detailed models of human cortical microcircuits, showing somatic placement of 1000 neurons in a 500x500x950 $\mu^{3}$ volume along layer 2/3 (250 - $1200 \mu \mathrm{m}$ below pia) and reconstructed morphologies used in the neuron models. (b - d) Temporally aligned multi-scale simulated signals: EEG (b) from scalp electrode directly above the microcircuit; LFP signal (c) recorded at the middle of L2/3 (depth of -725 $\mu \mathrm{m}$ ); Raster plot of spiking in different neurons in the microcircuit (d), color-coded according to neuron type. Neurons received background excitatory inputs to generate intrinsic circuit activity.

Baseline microcircuit activity was oscillatory, marked by synchronous spiking events (Fig 1d) and corresponding fluctuations in LFP and EEG signals (Fig 1b, c). We quantified oscillatory activity in the healthy microcircuits by calculating EEG power spectral density (PSD). The microcircuits EEG exhibited a peak in theta $(4-8 \mathrm{~Hz})$ and alpha $(8-12 \mathrm{~Hz})$ bands $(\mathrm{n}=60$ randomized microcircuits, Fig 2a) and a 1/f background trend (Fig 2a, inset). Peak frequency was $9.9 \pm 0.8 \mathrm{~Hz}$. We then calculated spectrograms of circuit activity to analyze the evolution of signal strength in the time-frequency domain. The spectrograms showed $34 \pm 5$ transient theta-alpha events per $28 \mathrm{~s}$ simulation, with average duration of $300 \pm 15 \mathrm{~ms}$ (Fig 2b). The circuit simulations thus reproduced several key temporal and spectral features of resting-state human EEG. Critically, these oscillatory properties were emergent, and were not explicitly optimized for, which therefore constitutes an important validation of the model and demonstration of its ability to capture key properties of human cortical L2/3 microcircuit dynamics. 

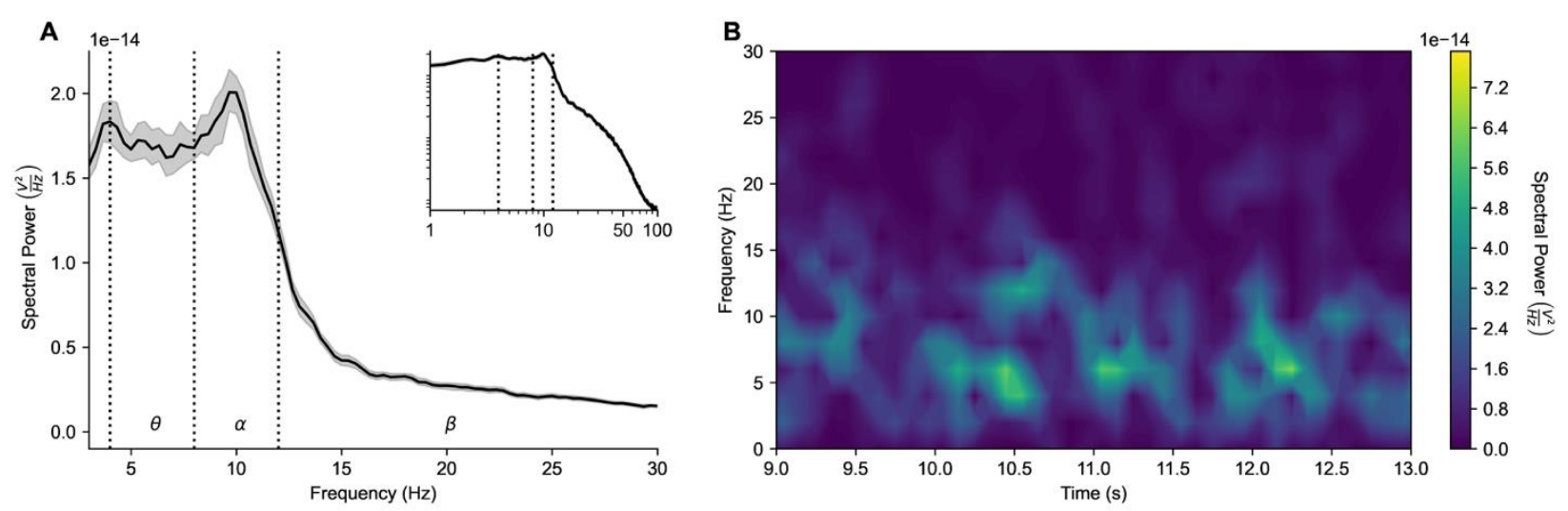

Figure 2. Models of human cortical microcircuits reproduce key features of resting-state EEG. (a) Power spectral density plot of circuit simulations $(n=60$ randomized microcircuits, bootstrapped mean and 95\% confidence intervals), exhibiting peak power in theta and alpha frequency bands. Inset - same power spectral density plot shown on log-log scale, illustrating the 1/f relationship between power and $\log$ frequency, inversely linear between $3-30 \mathrm{~Hz}$ with slope $-0.98 \pm 0.07$. Frequency bands are delimited by dotted lines. (b) Example spectrogram of simulated microcircuit EEG, exhibiting theta and alpha events.

\section{EEG biomarkers of reduced SST in depression microcircuits}

We compared simulated EEG in healthy versus depression microcircuits using our previous depression models, in which SST synaptic and tonic inhibition were reduced $(n=60$ randomized microcircuits, Fig 3a). The simulated EEG from depression microcircuits exhibited a prominent peak in theta and alpha bands $(4-12 \mathrm{~Hz})$ similarly to the healthy microcircuits, but there was a significantly increased power in these bands and in low-beta frequencies $(5-15 \mathrm{~Hz}, 25 \%$ increase on average, $p<0.05$, Cohen's $d=1.91$, Fig 3b). We decomposed EEG PSDs into aperiodic (Fig 3c) and periodic (Fig 3d) components, to compare the distinct functional components of the absolute PSDs. There was a $43 \%$ increase in aperiodic broadband power in depression compared to healthy microcircuits $(5-30 \mathrm{~Hz}, p<0.05$, Cohen's $d=5.6)$, and a $35 \%$ reduction in aperiodic exponent (healthy $=0.98 \pm 0.07$, depression $=0.64 \pm 0.07, p<0.05$, Cohen's $d=4.6$ ). The periodic component of the PSDs from depression microcircuits showed a $29 \%$ increase in theta power (5.6 $-8 \mathrm{~Hz}, p<0.05$, Cohen's $d=1.5)$, and a $63 \%$ increase in low-beta power $(12-15 \mathrm{~Hz}, p<0.05$, Cohen's $d=2.4$ ), but no significant change in alpha power compared to healthy microcircuits. Thus, the absolute power increase involved a combination of an aperiodic broadband component, and a periodic theta and low beta component. 
A

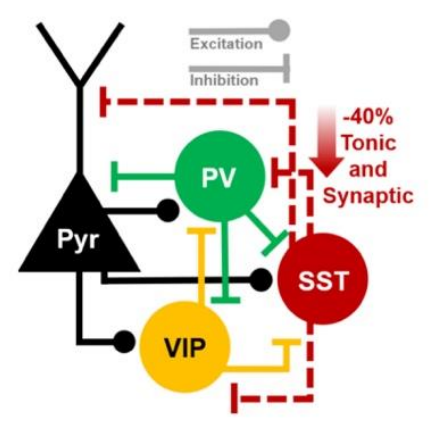

C

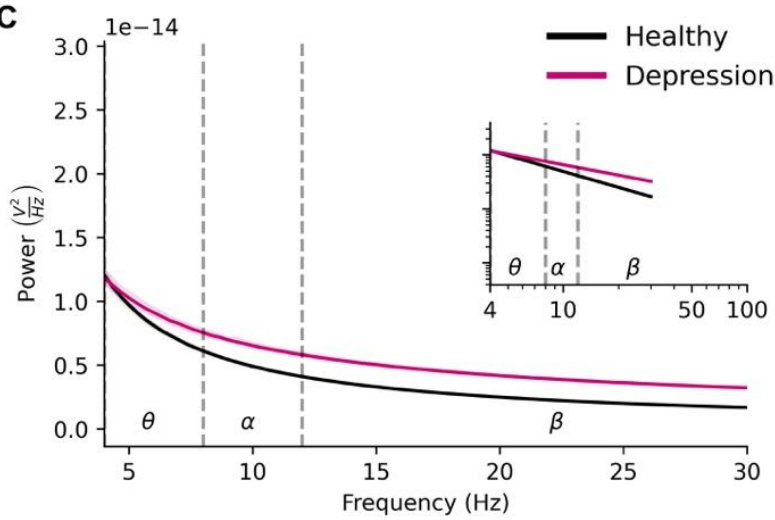

B

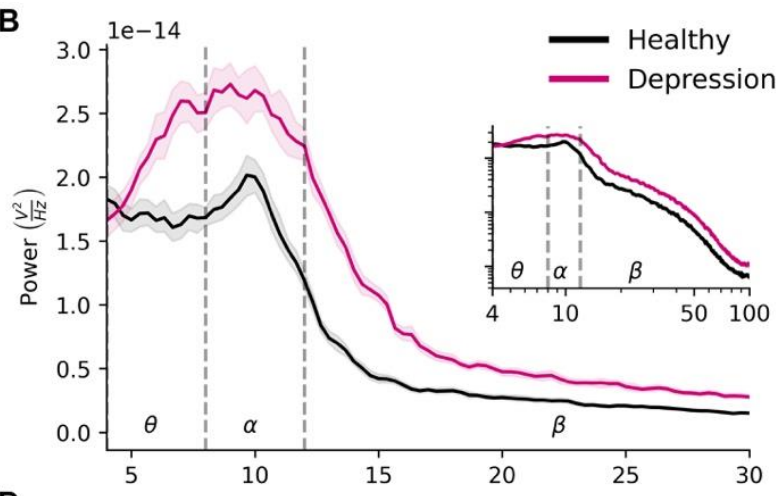

D

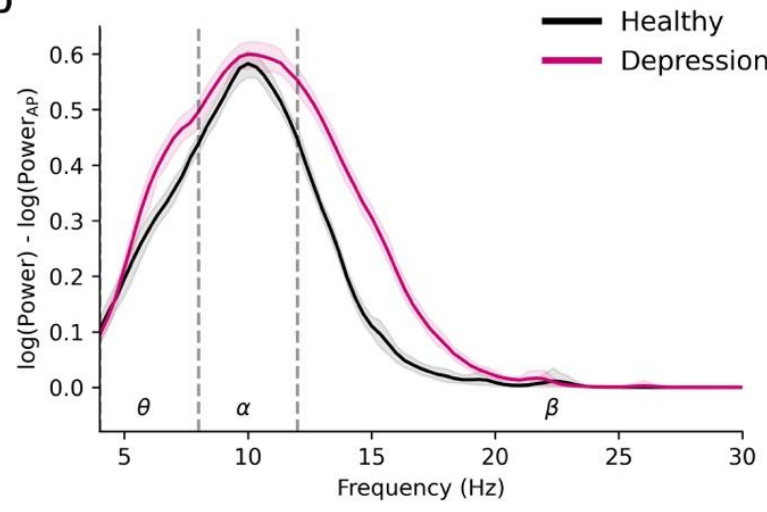

Figure 3. EEG signatures of reduced SST inhibition in depression microcircuit models. (a) Schematic connectivity diagram highlighting the key connections between different neuron types in the microcircuit. In the depression condition, dotted lines from the SST interneurons illustrate reduced synaptic and tonic inhibition onto different neuron types. (b) Power spectral density plot of simulated EEG from the healthy (black) and depression (magenta) microcircuit models ( $\mathrm{n}=60$ randomized microcircuits per condition, bootstrapped mean and 95\% confidence interval). (c) Fitted aperiodic component of the PSD. (d) Fitted periodic component of the PSD.

We identified relationships between neuronal spiking and EEG spectral changes in depression microcircuit models by determining the EEG phase preference of the neuronal populations. The EEG time series were bandpass filtered from $4-12 \mathrm{~Hz}$ since the periodic component of the PSDs showed equally prominent theta and alpha components (Fig 4d). Spike time was then converted to phase of EEG signal by temporally aligning the bandpass-filtered signal to the angle of its Hilbert transform (Fig 4a-c). In healthy microcircuits, spiking in all neuronal types exhibited preference to the phase preceding the trough of the EEG waveform (Rayleigh's $p$ $\left.<0.05 ; \mathrm{Pyr}=268.2 \pm 2.5^{\circ}, \mathrm{SST}=266.5 \pm 4.3^{\circ}, \mathrm{PV}=282.0 \pm 3.2^{\circ}, \mathrm{VIP}=289.6 \pm 2.0^{\circ}\right)$. Depression microcircuits followed a similar phase preference (Rayleigh's $p<0.05 ; \mathrm{Pyr}=281 \pm 2.2^{\circ}, \mathrm{SST}=$ $\left.279.5 \pm 5.0^{\circ}, \mathrm{PV}=293.1 \pm 3.0^{\circ}, \mathrm{VIP}=295.3 \pm 2.2^{\circ}\right)$, but there was a decrease in preference selectivity, as quantified by population spike concentrations about the preferred phase (kurtosis, $p$ 
$<0.05$ for all; $\%$ decrease: $\mathrm{Pyr}=12.9 \%, d=2.6 ; \mathrm{SST}=19.3 \%, d=2.0 ; \mathrm{PV}=21.3 \%, d=3.9, \mathrm{VIP}$ $=26.3 \%, d=5.5)$.

A

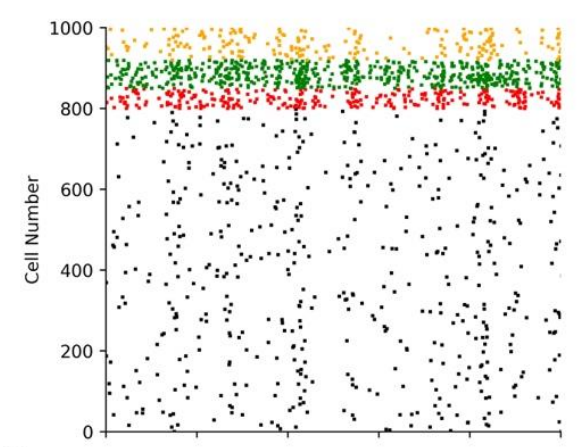

B

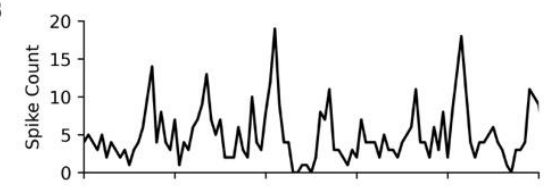

C

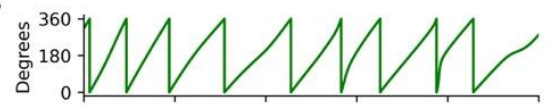

D

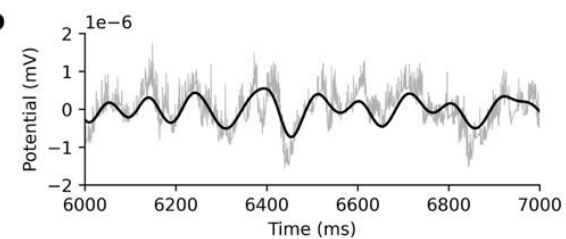

$\mathbf{E}$
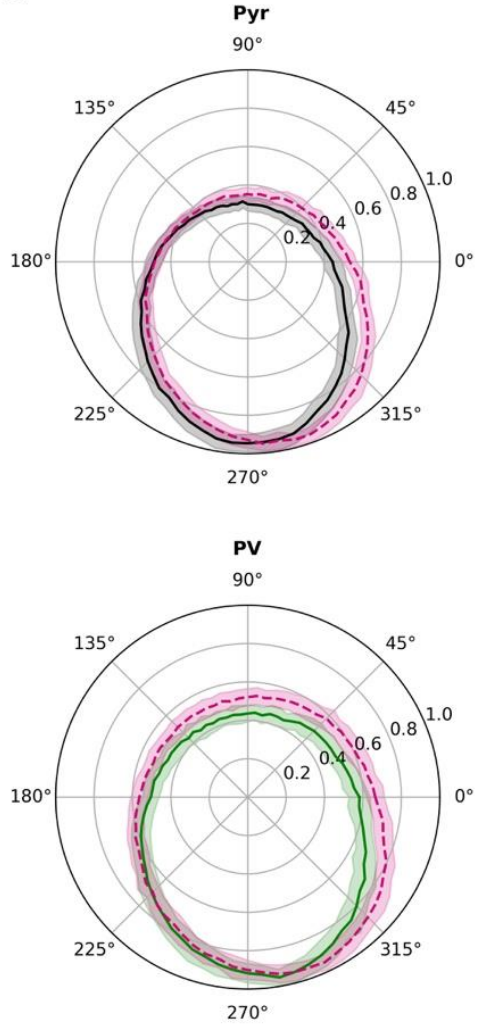

Figure 4. EEG phase preference of neuronal spiking in health and depression microcircuit models. (a-d) Temporally aligned simulated microcircuit signals used in calculating population phase-preference. (a) Raster plot of spiking in an example healthy microcircuit model. (b) Pyr population spike counts, in 10 ms bins. (c) Instantaneous phase of the theta-alpha bandpass filtered EEG signal, with trough corresponding to $0^{\circ}$. (d) Theta-alpha bandpass filtered EEG signal (black) and unfiltered EEG signal (gray). (e) Population spike counts relative to theta-alpha $(4-12 \mathrm{~Hz})$ bandpass filtered EEG from healthy (non-magenta) and depression microcircuits (magenta). Plots show bootstrapped mean and standard deviation normalized by peak spike count. Neuron type colors for the healthy microcircuits are as in Fig 1.

Lastly, we examined the spatial distribution of scalp-measured EEG signals generated by the microcircuit simulations using a realistic head model, and determined the distance at which it was indistinguishable from noise. We placed the simulated microcircuit dipoles into L2/3 of the dorsolateral PFC, at a location directly underneath 10-20 system electrode AF3 (Fig 5a), and used a forward solution based on a boundary-element model with realistic head geometry and conductivity to compute simulated EEG signals. The resulting time series were obtained at different electrode locations on the scalp (Fig 5b). Difference in theta-alpha power in depression versus healthy microcircuits showed a nonuniform decay over the scalp, which was steeper 
laterally compared to medially (c.f. contour lines in Fig 5c). A significant difference in power was only seen in electrodes corresponding to the ventral portion of the left dIPFC (AF3, the electrode above source) and the left orbitofrontal cortex (FP1 and FPz - 29mm and 46mm from source, respectively). The EEG biomarkers corresponding to microcircuit changes in depression were therefore mostly localized spatially.
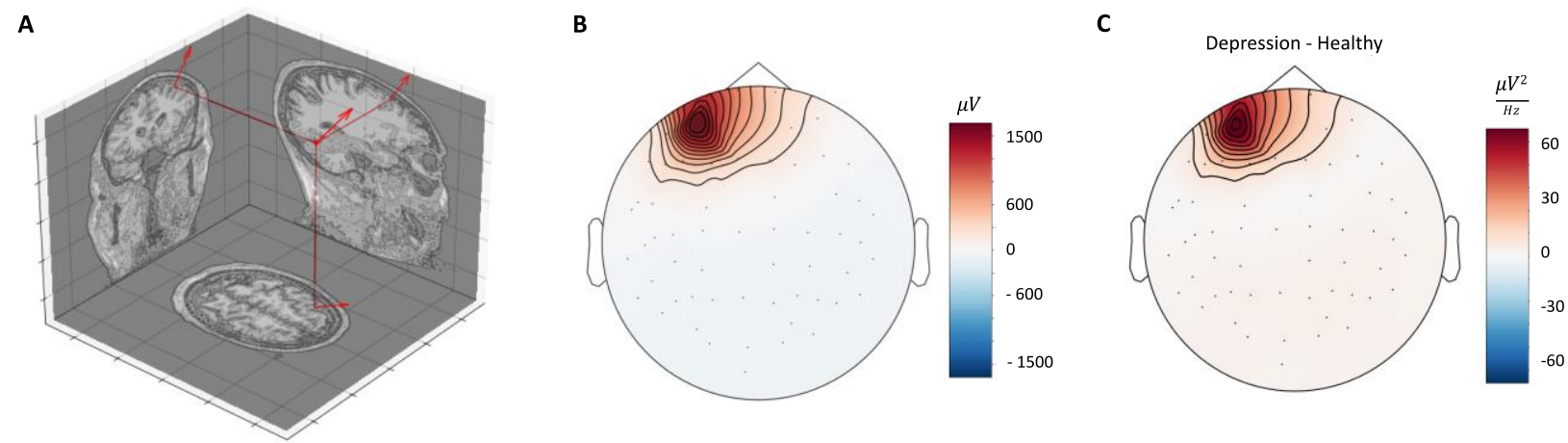

Figure 5: Topographic distribution of microcircuit EEG signal in a realistic head model. (a) Microcircuit dipole placement and orientation in the grey matter of the dlPFC in a head model, perpendicular to the cortical surface and in the middle of L2/3 (depth $-725 \mu \mathrm{m}$ ). (b) Corresponding average potential spread of the microcircuit EEG signal to the EEG electrodes across the head. (c) Distribution of the difference in spectral power for depression versus healthy simulated microcircuit signals for theta-alpha band $(4-12 \mathrm{~Hz})$.

\section{Discussion}

In this study, we identified the signatures of reduced cortical inhibition in depression on clinically relevant brain signals of resting-state activity using detailed models of human cortical microcircuits. Depression microcircuits with reduced SST interneuron inhibition showed an increase in absolute theta, alpha and low beta power, which involved a broadband increase together with a periodic increase in theta and low-beta activity. These signatures may be used as biomarkers to stratify depression subtypes corresponding to altered inhibition, which are particularly associated with treatment-resistant depression[41]. By simulating both spiking and EEG in detailed microcircuits, we showed that neuronal spiking had a marked preference for the phase preceding the trough of theta-alpha EEG band in both conditions. Our results provide mechanistic links between reduced SST inhibition and candidate biomarkers in EEG signals. These findings have multiple clinical applications, such as improving patient stratification and monitoring the effects of therapeutic compounds targeting SST interneuron inhibition. 
Increased theta, alpha, and low beta power in our simulated depression microcircuits are in agreement with several previous depression studies, and are correlated with diagnosis, severity, and treatment response [7,42-47]. Particularly, increased frontal theta power is seen in both the early and late stages of depression, indicating a consistent circuit mechanism underlying the pathology[43-46]. Further, increased frontal theta power is predictive of second line treatment response targeting cortical inhibition in treatment resistant depression [46]. Thus, our results provide support for reduced SST inhibition as a principal mechanism of treatment-resistant depression, and show that it could be detectible in its early stages by increased low frequency power[43,45,48-50]. Future studies relating our simulated EEG biomarkers to experimental EEG and depression features will further establish the link to disease stage, subtype and severity. The increased aperiodic broadband power and decreased aperiodic slope in our depression microcircuits are consistent with the respective effects of increased excitation-inhibition ratio and increased baseline activity [51-53], which in our simulations resulted from reduced SST inhibition. Increased rhythmic theta and low beta activity, but not alpha, may explain a lack of change in relative alpha power seen previously and lend more functional significance to theta and low beta frequencies[54-57]. As previous studies have shown that theta waves modulate network excitability[58], the increased theta activity in depression microcircuits may have further functional consequences via altering cortical dynamics and processing. In summary, our results show that altered inhibition in microscale brain circuits in depression is reflected in key features of the EEG spectral density, which can be used to better stratify depression subtype and severity.

Our biophysical models of human cortical microcircuits reproduce the two most ubiquitous features of human resting state EEG: a prominent low-frequency $(4-12 \mathrm{~Hz})$ peak power and 1/f aperiodic trend [59,60]. In experimental EEG recordings, this low-frequency peak is accentuated when subjects are relaxed with their eyes closed and has the highest test-retest reliability of all spectral features[61]. While recurrent rhythmic activity generated within cortico-thalamic loops is believed to contribute strongly to scalp-measured EEG alpha, cortico-cortical interactions have also been shown to generate alpha activity in top-down processing[62]. Further, it has been shown that cortical Pyr neurons significantly contribute to and sustain theta and alpha-phasic firing due to intrinsic properties and recurrent activity[62-64]. The key features of human EEG reproduced by our microcircuit models were not explicitly constrained for, but rather emerged from the interaction of human neuronal electrical properties, synaptic connectivity, and baseline firing rates. 
This provides a validation of the models and indicates that they contain circuit motifs responsible for generating realistic microcircuit activity. It will be of interest in future studies to use the models for further elucidating the cellular mechanisms of oscillatory generation, especially in brain states other than rest and during cortical processing, which involve other frequency bands. As our models capture the key spectral EEG features seen in vivo, the simulated EEG signal magnitude can also be related to that of the recorded EEG by scaling to account for the difference between the number of neurons in our models compared to the millions of neurons that generate the recorded EEG at a given electrode[40,65].

We simulated surface EEG using cortical L2/3 microcircuits, as these layers are the primary contributors to the EEG signal[66]. As new human cellular data becomes available, future models that also include deeper layers $(4-6)$ could further refine the estimated EEG signal and the oscillatory dynamics mediated by interactions between deep and superficial layers[35]. Through coupling the biophysical simulations with a forward solution using a realistic head model we showed that EEG signals would be fairly localized to the source and neighboring electrodes. These computational simulations can serve to further study source localization in interpreting EEG recordings, improve inverse solutions[67], give greater physiological interpretability to statistical decomposition techniques such as independent component analysis[68] and s/eLORETA[69]. This is especially relevant in depression, where distinct brain sub-regions both serve as targets for treatment interventions, such as the lateral/medial PFC and lateral orbitofrontal cortex[70-73], and exhibit different spectral power changes in relation to diagnosing depression stage and type[45]. While we modeled and studied a single cortical region at the microcircuit scale, future studies could simulate several microcircuits to examine how the altered circuit inhibition mechanisms affect multi-regional interactions in depression[8].

Using detailed multi-scale models of human cortical microcircuits, we were able to characterize the EEG and spike correlates of reduced SST inhibition in depression. Previous modeling studies have shown that reduced SST inhibition in depression impairs stimulus processing due to increased baseline activity and noise[24]. Our spiking-EEG phase analysis indicates that during the peak of theta-alpha phase, stimulus detection would be further impaired than at the down-phase. Our models can serve to further identify candidate EEG biomarkers of the diverse mechanisms of depression, e.g. the possible altered PV vs. SST inhibition[8], pyramidal cell atrophy and synapses loss[74-78]. Furthermore, the computational models we have developed 
provide a powerful tool to identify the EEG biomarkers of novel therapeutic compounds and treatments for depression[17,79] via in-silico simulations to improve treatment monitoring. Finally, our models and methodology may further serve to identify EEG biomarkers of altered cellular and circuit mechanism in other neurological diseases, such as epilepsy and schizophrenia[39,80-83].

\section{Methods}

Human cortical microcircuit models. We used our previous models of human cortical L2/3 microcircuits[24], consisting of 1000 neurons distributed in a 500x500x950 $\mu^{3}$ volume (250 to $1200 \mu \mathrm{m}$ below pia[84]). The model microcircuits included the four key neuron types in cortical L2/3: Pyramidal (Pyr), Somatostatin-expressing (SST), Parvalbumin-expressing (PV), and Vasoactive Intestinal Peptide-expressing (VIP) neurons. The proportions of the neuron types were: $80 \% \mathrm{Pyr}, 5 \% \mathrm{SST}, 7 \% \mathrm{PV}$, and 8\% VIP in accordance with relative L2/3 neuron densities[85,86] and RNA-seq data[87,88]. The models were simulated using NEURON[89] and LFPy[38].

Resting-state activity simulations. We simulated eyes-closed resting-state by injecting the microcircuit models with background excitatory input representing cortical and thalamic drive, as used previously[24]. The background input was generated by random Orstein-Uhlenbeck (OU) point processes[90] placed on all neurons at the halfway point of each dendritic arbor, and 5 additional OU processes placed along the apical trunk of Pyr neurons in equal intervals, from 10\% to $90 \%$ apical dendritic length. This enabled the circuit to generate recurrent activity with neuronal firing rates as measured in vivo[23,24,91,92]. For both healthy and depression microcircuit models (see below) we simulated 60 randomized microcircuits, for 28 seconds each.

Depression models. We used previous models of depression microcircuits[24], with $40 \%$ reduced synaptic and tonic inhibition from SST interneurons onto all other neurons, in accordance with gene expression studies in SST interneurons in post-mortem brains from depression patients[13]. For Pyr neurons, we decreased apical tonic inhibition by $40 \%$. For each interneuron, we decreased the relative SST contribution to tonic inhibition by $40 \%$. 
Simulated microcircuit EEG. We simulated layer-averaged dipole moments together with neuronal activity using LFPy. Corresponding EEG time series was generated using a four-sphere volume conductor model that assumes homogeneous, isotropic, and linear (frequency independent) conductivity. The radii of the four spheres representing grey matter, cerebrospinal fluid, skull, and scalp were $79 \mathrm{~mm}, 80 \mathrm{~mm}, 85 \mathrm{~mm}$, and $90 \mathrm{~mm}$, respectively. The conductivity for each sphere was $0.047 \mathrm{~S} / \mathrm{m}, 1.71 \mathrm{~S} / \mathrm{m}, 0.02 \mathrm{~S} / \mathrm{m}$, and $0.41 \mathrm{~S} / \mathrm{m}$, respectively[93]. A fixed dipole origin was placed at the midpoint of L2/3 $(-725 \mu \mathrm{m})$, and the EEG signal was obtained via forward solution for an EEG electrode placed on the scalp surface. EEG time series were lowpass filtered at $130 \mathrm{~Hz}$. EEG power spectral density (PSD) and spectrograms were calculated using Welch's method[94], with a 3s second Hanning window for PSD, and 0.5s Hanning window for spectrograms. Both analyses used $80 \%$ window overlap.

EEG periodic and aperiodic components. We decomposed EEG PSDs into periodic and aperiodic (broadband) components using tools from the FOOOF library[51]. The aperiodic component of the PSD was a 1/f function, defined by a vertical offset and exponent parameter. Periodic components representing putative oscillations were derived using up to 4 Gaussians, defined by center frequency (mean), bandwidth (variance), and power (height).

Population spiking phase preference. We calculated the instantaneous phase of EEG using tools from the SciPy[95] and NumPy[96] Python libraries. The EEG time series was bandpass filtered from $4-12 \mathrm{~Hz}$, with $2 \mathrm{~Hz}$ padding on either end to avoid edge effects. A second order Butterworth filter was used to acquire filter coefficients, which were then passed to a linear digital filter applied forward and backwards to eliminate phase lag. The angle of the bandpass-filtered Hilbert transform was calculated and shifted by $180^{\circ}$ to obtain the instantaneous phase of the signal with trough and peak corresponding to $0^{\circ}$ and $180^{\circ}$, respectively. For each of the four neuron populations (Pyr, SST, PV, VIP), spike times were aggregated into a population spike vector and converted to corresponding phase of EEG. Spike phases were binned into $5^{\circ}$ bins and the counts were summated over all circuits in each condition. To compare spike preference between conditions, bin counts were normalized by the maximal count. 
Realistic head source modelling. The $x-y-z$ components of microcircuit dipole moments generated from LFPy were imported into MNE[97] and downsampled to a $400 \mathrm{~Hz}$ sampling rate. The dipole was placed within L2/3 of the left dorsolateral PFC (MNI 30, -36, -42)[98], which has been implicated in depression[99]. We solved the forward solution using a three-layer boundary element model, as implemented in LFPy, corresponding to the inner skull, outer skull, and scalp with conductivities of $0.3 \mathrm{~S} / \mathrm{m}, 0.006 \mathrm{~S} / \mathrm{m}$ and $0.3 \mathrm{~S} / \mathrm{m}$, respectively. The resulting potential was calculated for a standard 60-channel simulated EEG electrode array.

Statistical tests. We used two-sample t-test to determine statistical significance where appropriate. We calculated Cohen's $d$ to show effect size. We used Raileigh's test of non-uniformity to determine if populations had a non-uniform phase preference, and kurtosis to quantify variance around the preferred mean angle.

Data availability statement. All models and code will be available online upon publication.

\section{Acknowledgements}

FM, JG, and EH thank the Krembil Foundation and the Ontario Graduate Scholarship for funding support. FM and EH were also supported by a stipend award from the Department of Physiology at University of Toronto. 


\section{References}

1. Chiu M, Lebenbaum M, Cheng J, de Oliveira C, Kurdyak P. The direct healthcare costs associated with psychological distress and major depression: A population-based cohort study in Ontario, Canada. PLoS ONE. 2017;12. doi:10.1371/journal.pone.0184268

2. Verduijn J, Milaneschi Y, Schoevers RA, van Hemert AM, Beekman ATF, Penninx BWJH. Pathophysiology of major depressive disorder: mechanisms involved in etiology are not associated with clinical progression. Transl Psychiatry. 2015;5: e649-e649. doi:10.1038/tp.2015.137

3. Prins J, Olivier B, Korte SM. Triple reuptake inhibitors for treating subtypes of major depressive disorder: the monoamine hypothesis revisited. Expert Opin Investig Drugs. 2011;20: 11071130. doi:10.1517/13543784.2011.594039

4. Al-Harbi KS. Treatment-resistant depression: therapeutic trends, challenges, and future directions. Patient Prefer Adherence. 2012;6: 369-388. doi:10.2147/PPA.S29716

5. Fitzgerald PB, Hoy KE, Anderson RJ, Daskalakis ZJ. a study of the pattern of response to rTMS treatment in depression. Depress Anxiety. 2016;33: 746-753. doi:10.1002/da.22503

6. Olbrich S, Arns M. EEG biomarkers in major depressive disorder: Discriminative power and prediction of treatment response. Int Rev Psychiatry. 2013;25: 604-618. doi:10.3109/09540261.2013.816269

7. Jaworska N, Blier P, Fusee W, Knott V. Alpha power, alpha asymmetry and anterior cingulate cortex activity in depressed males and females. J Psychiatr Res. 2012;46: 1483-1491. doi:10.1016/j.jpsychires.2012.08.003

8. Northoff G, Sibille E. Why are cortical GABA neurons relevant to internal focus in depression? A cross-level model linking cellular, biochemical and neural network findings. Mol Psychiatry. 2014;19: 966-977. doi:10.1038/mp.2014.68

9. Prévot T, Sibille E. Altered GABA-mediated information processing and cognitive dysfunctions in depression and other brain disorders. Mol Psychiatry. 2021;26: 151-167. doi:10.1038/s41380-020-0727-3

10. Fuchs T, Jefferson SJ, Hooper A, Yee P-H, Maguire J, Luscher B. Disinhibition of somatostatin-positive GABAergic interneurons results in an anxiolytic and antidepressantlike brain state. Mol Psychiatry. 2017;22: 920-930. doi:10.1038/mp.2016.188

11. Lin L-C, Sibille E. Reduced brain somatostatin in mood disorders: a common pathophysiological substrate and drug target? Front Pharmacol. 2013;4: 110. doi:10.3389/fphar.2013.00110

12. Fee C, Prevot TD, Misquitta K, Knutson DE, Li G, Mondal P, et al. Behavioral deficits induced by somatostatin-positive GABA neuron silencing are rescued by alpha 5 GABA-A receptor potentiation. Int J Neuropsychopharmacol. 2021. doi:10.1093/ijnp/pyab002 
13. Seney ML, Tripp A, McCune S, Lewis D, Sibille E. Laminar and Cellular Analyses of Reduced Somatostatin Gene Expression in the Subgenual Anterior Cingulate Cortex in Major Depression. Neurobiol Dis. 2015;73: 213-219. doi:10.1016/j.nbd.2014.10.005

14. Sibille E, Morris HM, Kota RS, Lewis DA. GABA-related transcripts in the dorsolateral prefrontal cortex in mood disorders. Int J Neuropsychopharmacol. 2011;14: 721-734. doi:10.1017/S1461145710001616

15. Tripp A, Kota RS, Lewis DA, Sibille E. Reduced somatostatin in subgenual anterior cingulate cortex in major depression. Neurobiol Dis. 2011;42: 116-124. doi:10.1016/j.nbd.2011.01.014

16. Guilloux J-P, Douillard-Guilloux G, Kota R, Wang X, Gardier A, Martinowich K, et al. Molecular evidence for BDNF- and GABA-related dysfunctions in the amygdala of female subjects with Major Depression. Mol Psychiatry. 2012;17: 1130-1142. doi:10.1038/mp.2011.113

17. Prevot TD, Li G, Vidojevic A, Misquitta KA, Fee C, Santrac A, et al. Novel BenzodiazepineLike Ligands with Various Anxiolytic, Antidepressant, or Pro-Cognitive Profiles. Complex Psychiatry. 2019;5: 84-97. doi:10.1159/000496086

18. Olejniczak P. Neurophysiologic basis of EEG. J Clin Neurophysiol Off Publ Am Electroencephalogr Soc. 2006;23: 186-189. doi:10.1097/01.wnp.0000220079.61973.6c

19. Tremblay R, Lee S, Rudy B. GABAergic interneurons in the neocortex: From cellular properties to circuits. Neuron. 2016;91: 260-292. doi:10.1016/j.neuron.2016.06.033

20. Ali AB, Thomson AM. Synaptic alpha 5 subunit-containing GABAA receptors mediate IPSPs elicited by dendrite-preferring cells in rat neocortex. Cereb Cortex N Y N 1991. 2008;18: 1260-1271. doi:10.1093/cercor/bhm160

21. Silberberg G, Markram H. Disynaptic Inhibition between Neocortical Pyramidal Cells Mediated by Martinotti Cells. Neuron. 2007;53: 735-746. doi:10.1016/j.neuron.2007.02.012

22. Obermayer J, Heistek TS, Kerkhofs A, Goriounova NA, Kroon T, Baayen JC, et al. Lateral inhibition by Martinotti interneurons is facilitated by cholinergic inputs in human and mouse neocortex. Nat Commun. 2018;9: 4101. doi:10.1038/s41467-018-06628-w

23. Gentet LJ, Kremer Y, Taniguchi H, Huang ZJ, Staiger JF, Petersen CCH. Unique functional properties of somatostatin-expressing GABAergic neurons in mouse barrel cortex. Nat Neurosci. 2012;15: 607-612. doi:10.1038/nn.3051

24. Yao HK, Guet-McCreight A, Mazza F, Chameh HM, Prevot TD, Griffiths J, et al. Reduced inhibition in depression impairs stimulus processing in human cortical microcircuits. bioRxiv. 2021; 2021.02.17.431698. doi:10.1101/2021.02.17.431698 
25. Chen G, Zhang Y, Li X, Zhao X, Ye Q, Lin Y, et al. Distinct Inhibitory Circuits Orchestrate Cortical beta and gamma Band Oscillations. Neuron. 2017;96: 1403-1418.e6. doi:10.1016/j.neuron.2017.11.033

26. Huang P, Xiang X, Chen X, Li H. Somatostatin Neurons Govern Theta Oscillations Induced by Salient Visual Signals. Cell Rep. 2020;33: 108415. doi:10.1016/j.celrep.2020.108415

27. Funk CM, Peelman K, Bellesi M, Marshall W, Cirelli C, Tononi G. Role of SomatostatinPositive Cortical Interneurons in the Generation of Sleep Slow Waves. J Neurosci. 2017;37: 9132-9148. doi:10.1523/JNEUROSCI.1303-17.2017

28. Jiang H, Popov T, Jylänki P, Bi K, Yao Z, Lu Q, et al. Predictability of depression severity based on posterior alpha oscillations. Clin Neurophysiol Off J Int Fed Clin Neurophysiol. 2016;127: 2108-2114. doi:10.1016/j.clinph.2015.12.018

29. Dehghani N, Peyrache A, Telenczuk B, Le Van Quyen M, Halgren E, Cash SS, et al. Dynamic Balance of Excitation and Inhibition in Human and Monkey Neocortex. Sci Rep. 2016;6: 23176. doi:10.1038/srep23176

30. Quyen MLV, Muller LE, Telenczuk B, Halgren E, Cash S, Hatsopoulos NG, et al. Highfrequency oscillations in human and monkey neocortex during the wake-sleep cycle. Proc Natl Acad Sci. 2016;113: 9363-9368. doi:10.1073/pnas.1523583113

31. Gardner RJ, Hughes SW, Jones MW. Differential Spike Timing and Phase Dynamics of Reticular Thalamic and Prefrontal Cortical Neuronal Populations during Sleep Spindles. J Neurosci. 2013;33: 18469-18480. doi:10.1523/JNEUROSCI.2197-13.2013

32. Averkin RG, Szemenyei V, Bordé S, Tamás G. Identified Cellular Correlates of Neocortical Ripple and High-Gamma Oscillations during Spindles of Natural Sleep. Neuron. 2016;92: 916-928. doi:10.1016/j.neuron.2016.09.032

33. McGinn RJ, Valiante TA. Phase-Amplitude Coupling and Interlaminar Synchrony Are Correlated in Human Neocortex. J Neurosci. 2014;34: 15923-15930. doi:10.1523/JNEUROSCI.2771-14.2014

34. Fedele T, Boran E, Chirkov V, Hilfiker P, Grunwald T, Stieglitz L, et al. Dataset of spiking and LFP activity invasively recorded in the human amygdala during aversive dynamic stimuli. Sci Data. 2021;8: 9. doi:10.1038/s41597-020-00790-x

35. Florez CM, McGinn RJ, Lukankin V, Marwa I, Sugumar S, Dian J, et al. In vitro recordings of human neocortical oscillations. Cereb Cortex N Y N 1991. 2015;25: 578-597. doi:10.1093/cercor/bht235

36. Seeman SC, Campagnola L, Davoudian PA, Hoggarth A, Hage TA, Bosma-Moody A, et al. Sparse recurrent excitatory connectivity in the microcircuit of the adult mouse and human cortex. Slutsky I, Marder E, Sjöström PJ, editors. eLife. 2018;7: e37349. doi:10.7554/eLife.37349 
37. Chameh HM, Rich S, Wang L, Chen F-D, Zhang L, Carlen PL, et al. Sag currents are a major contributor to human pyramidal cell intrinsic differences across cortical layers. bioRxiv. 2020; 748988. doi:10.1101/748988

38. Hagen E, Næss S, Ness TV, Einevoll GT. Multimodal modeling of neural network activity: computing LFP, ECoG, EEG and MEG signals with LFPy2.0. bioRxiv. 2018; 281717. doi: $10.1101 / 281717$

39. Mäki-Marttunen T, Krull F, Bettella F, Hagen E, Næss S, Ness TV, et al. Alterations in Schizophrenia-Associated Genes Can Lead to Increased Power in Delta Oscillations. Cereb Cortex. 2019;29: 875-891. doi:10.1093/cercor/bhy291

40. Jones SR, Pritchett DL, Sikora MA, Stufflebeam SM, Hämäläinen M, Moore CI. Quantitative analysis and biophysically realistic neural modeling of the MEG mu rhythm: rhythmogenesis and modulation of sensory-evoked responses. J Neurophysiol. 2009;102: 3554-3572. doi:10.1152/jn.00535.2009

41. Fee C, Banasr M, Sibille E. Somatostatin-Positive Gamma-Aminobutyric Acid Interneuron Deficits in Depression: Cortical Microcircuit and Therapeutic Perspectives. Biol Psychiatry. 2017;82: 549-559. doi:10.1016/j.biopsych.2017.05.024

42. Fernández-Palleiro $\mathrm{P}$, Rivera-Baltanás T, Rodrigues-Amorim D, Fernández-Gil S, del Carmen Vallejo-Curto M, Álvarez-Ariza M, et al. Brainwaves Oscillations as a Potential Biomarker for Major Depression Disorder Risk. Clin EEG Neurosci. 2020;51: 3-9. doi:10.1177/1550059419876807

43. Newson JJ, Thiagarajan TC. EEG Frequency Bands in Psychiatric Disorders: A Review of Resting State Studies. Front Hum Neurosci. 2018;12: 521. doi:10.3389/fnhum.2018.00521

44. Arns M, Etkin A, Hegerl U, Williams LM, DeBattista C, Palmer DM, et al. Frontal and rostral anterior cingulate (rACC) theta EEG in depression: implications for treatment outcome? Eur Neuropsychopharmacol J Eur Coll Neuropsychopharmacol. 2015;25: 1190-1200. doi:10.1016/j.euroneuro.2015.03.007

45. Grin-Yatsenko VA, Baas I, Ponomarev VA, Kropotov JD. Independent component approach to the analysis of EEG recordings at early stages of depressive disorders. Clin Neurophysiol. 2010;121: 281-289. doi:10.1016/j.clinph.2009.11.015

46. Bailey NW, Hoy KE, Rogasch NC, Thomson RH, McQueen S, Elliot D, et al. Responders to rTMS for depression show increased fronto-midline theta and theta connectivity compared to non-responders. Brain Stimulat. 2018;11: 190-203. doi:10.1016/j.brs.2017.10.015

47. Bruder GE, Sedoruk JP, Stewart JW, McGrath PJ, Quitkin FM, Tenke CE. Electroencephalographic Alpha Measures Predict Therapeutic Response to a Selective Serotonin Reuptake Inhibitor Antidepressant: Pre- and Post-Treatment Findings. Biol Psychiatry. 2008;63: 1171-1177. doi:10.1016/j.biopsych.2007.10.009 
48. de Aguiar Neto FS, Rosa JLG. Depression biomarkers using non-invasive EEG: A review. Neurosci Biobehav Rev. 2019;105: 83-93. doi:10.1016/j.neubiorev.2019.07.021

49. Olbrich S, Arns M. EEG biomarkers in major depressive disorder: discriminative power and prediction of treatment response. Int Rev Psychiatry Abingdon Engl. 2013;25: 604-618. doi:10.3109/09540261.2013.816269

50. Smith DF. Quest for Biomarkers of Treatment-Resistant Depression: Shifting the Paradigm Toward Risk. Front Psychiatry. 2013;4. doi:10.3389/fpsyt.2013.00057

51. Donoghue T, Haller M, Peterson EJ, Varma P, Sebastian P, Gao R, et al. Parameterizing neural power spectra into periodic and aperiodic components. Nat Neurosci. 2020;23: 16551665. doi:10.1038/s41593-020-00744-X

52. Gao R, Peterson EJ, Voytek B. Inferring synaptic excitation/inhibition balance from field potentials. NeuroImage. 2017;158: 70-78. doi:10.1016/j.neuroimage.2017.06.078

53. Podvalny E, Noy N, Harel M, Bickel S, Chechik G, Schroeder CE, et al. A unifying principle underlying the extracellular field potential spectral responses in the human cortex. J Neurophysiol. 2015;114: 505-519. doi:10.1152/jn.00943.2014

54. Knott V, Mahoney C, Kennedy S, Evans K. EEG power, frequency, asymmetry and coherence in male depression. Psychiatry Res Neuroimaging. 2001;106: 123-140. doi:10.1016/S0925-4927(00)00080-9

55. Morgan ML, Witte EA, Cook IA, Leuchter AF, Abrams M, Siegman B. Influence of age, gender, health status, and depression on quantitative EEG. Neuropsychobiology. 2005;52: 71-76. doi:10.1159/000086608

56. Korb AS, Cook IA, Hunter AM, Leuchter AF. Brain electrical source differences between depressed subjects and healthy controls. Brain Topogr. 2008;21: 138-146. doi:10.1007/s10548-008-0070-5

57. Cook IA, Hunter AM, Korb AS, Leuchter AF. Do prefrontal midline electrodes provide unique neurophysiologic information in Major Depressive Disorder? J Psychiatr Res. 2014;53: 69-75. doi:10.1016/j.jpsychires.2014.01.018

58. Buzsáki G. Theta oscillations in the hippocampus. Neuron. 2002;33: 325-340. doi:10.1016/s0896-6273(02)00586-x

59. Nunez PL, Srinivasan R. Electric fields of the brain: the neurophysics of EEG. 2nd ed. Oxford ; New York: Oxford University Press; 2006.

60. Ouyang G, Hildebrandt A, Schmitz F, Herrmann CS. Decomposing alpha and 1/f brain activities reveals their differential associations with cognitive processing speed. Neuroimage. 2020;205. doi:10.1016/j.neuroimage.2019.116304 
61. Gasser T, Bächer P, Steinberg H. Test-retest reliability of spectral parameters of the EEG. Electroencephalogr Clin Neurophysiol. 1985;60: 312-319. doi:10.1016/00134694(85)90005-7

62. Halgren M, Ulbert I, Bastuji H, Fabó D, Erőss L, Rey M, et al. The generation and propagation of the human alpha rhythm. Proc Natl Acad Sci. 2019;116: 23772-23782. doi:10.1073/pnas.1913092116

63. Silva LR, Amitai Y, Connors BW. Intrinsic oscillations of neocortex generated by layer 5 pyramidal neurons. Science. 1991;251: 432-435. doi:10.1126/science.1824881

64. Connors BW, Gutnick MJ. Intrinsic firing patterns of diverse neocortical neurons. Trends Neurosci. 1990;13: 99-104. doi:10.1016/0166-2236(90)90185-D

65. Murakami S, Okada Y. Contributions of principal neocortical neurons to magnetoencephalography and electroencephalography signals. J Physiol. 2006;575: 925936. doi:10.1113/jphysiol.2006.105379

66. Buzsáki G, Anastassiou CA, Koch C. The origin of extracellular fields and currents--EEG, ECoG, LFP and spikes. Nat Rev Neurosci. 2012;13: 407-420. doi:10.1038/nrn3241

67. Grech R, Cassar T, Muscat J, Camilleri KP, Fabri SG, Zervakis M, et al. Review on solving the inverse problem in EEG source analysis. J NeuroEngineering Rehabil. 2008;5: 25. doi:10.1186/1743-0003-5-25

68. Makeig S, Bell AJ, Jung T-P, Sejnowski TJ. Independent Component Analysis of Electroencephalographic Data. : 7.

69. Pascual-Marqui RD, Lehmann D, Koukkou M, Kochi K, Anderer P, Saletu B, et al. Assessing interactions in the brain with exact low-resolution electromagnetic tomography. Philos Trans R Soc Math Phys Eng Sci. 2011;369: 3768-3784. doi:10.1098/rsta.2011.0081

70. Feffer K, Fettes P, Giacobbe P, Daskalakis ZJ, Blumberger DM, Downar J. 1Hz rTMS of the right orbitofrontal cortex for major depression: Safety, tolerability and clinical outcomes. Eur Neuropsychopharmacol J Eur Coll Neuropsychopharmacol. 2018;28: 109-117. doi:10.1016/j.euroneuro.2017.11.011

71. Li Y, Wang L, Jia M, Guo J, Wang H, Wang M. The effects of high-frequency rTMS over the left DLPFC on cognitive control in young healthy participants. PLoS ONE. 2017;12. doi:10.1371/journal.pone.0179430

72. Bakker N, Shahab S, Giacobbe P, Blumberger DM, Daskalakis ZJ, Kennedy SH, et al. rTMS of the Dorsomedial Prefrontal Cortex for Major Depression: Safety, Tolerability, Effectiveness, and Outcome Predictors for $10 \mathrm{~Hz}$ Versus Intermittent Theta-burst Stimulation. Brain Stimulat. 2015;8: 208-215. doi:10.1016/j.brs.2014.11.002 
73. Fettes P, Schulze L, Downar J. Cortico-Striatal-Thalamic Loop Circuits of the Orbitofrontal Cortex: Promising Therapeutic Targets in Psychiatric Illness. Front Syst Neurosci. 2017;11. doi:10.3389/fnsys.2017.00025

74. Radley JJ, Rocher AB, Miller M, Janssen WGM, Liston C, Hof PR, et al. Repeated stress induces dendritic spine loss in the rat medial prefrontal cortex. Cereb Cortex N Y N 1991. 2006;16: 313-320. doi:10.1093/cercor/bhi104

75. Radley JJ, Sisti HM, Hao J, Rocher AB, McCall T, Hof PR, et al. Chronic behavioral stress induces apical dendritic reorganization in pyramidal neurons of the medial prefrontal cortex. Neuroscience. 2004;125: 1-6. doi:10.1016/j.neuroscience.2004.01.006

76. Koleske AJ. Molecular mechanisms of dendrite stability. Nat Rev Neurosci. 2013;14: 536550. doi:10.1038/nrn3486

77. Oh H, Piantadosi SC, Rocco BR, Lewis DA, Watkins SC, Sibille E. The Role of Dendritic Brain-Derived Neurotrophic Factor Transcripts on Altered Inhibitory Circuitry in Depression. Biol Psychiatry. 2019;85: 517-526. doi:10.1016/j.biopsych.2018.09.026

78. Shansky RM, Hamo C, Hof PR, McEwen BS, Morrison JH. Stress-induced dendritic remodeling in the prefrontal cortex is circuit specific. Cereb Cortex N Y N 1991. 2009;19: 2479-2484. doi:10.1093/cercor/bhp003

79. Krystal JH, Abdallah CG, Sanacora G, Charney DS, Duman RS. Ketamine: A Paradigm Shift for Depression Research and Treatment. Neuron. 2019;101: 774-778. doi:10.1016/j.neuron.2019.02.005

80. Avissar M, Xie S, Vail B, Lopez-Calderon J, Wang Y, Javitt DC. Meta-analysis of mismatch negativity to simple versus complex deviants in schizophrenia. Schizophr Res. 2018;191: 2534. doi:10.1016/j.schres.2017.07.009

81. Zhdanov A, Atluri S, Wong W, Vaghei Y, Daskalakis ZJ, Blumberger DM, et al. Use of Machine Learning for Predicting Escitalopram Treatment Outcome From Electroencephalography Recordings in Adult Patients With Depression. JAMA Netw Open. 2020;3: e1918377. doi:10.1001/jamanetworkopen.2019.18377

82. Ibrahim GM, Wong SM, Anderson RA, Singh-Cadieux G, Akiyama T, Ochi A, et al. Dynamic modulation of epileptic high frequency oscillations by the phase of slower cortical rhythms. Exp Neurol. 2014;251: 30-38. doi:10.1016/j.expneurol.2013.10.019

83. Udupa K, Tai P, Saha U, Wennberg R, Valiante T, Andrade D, et al. Theta burst transcranial magnetic stimulation to induce seizures in an epilepsy monitoring unit. Brain Stimul Basic Transl Clin Res Neuromodulation. 2020;13: 1800-1802. doi:10.1016/j.brs.2020.10.009

84. Mohan H, Verhoog MB, Doreswamy KK, Eyal G, Aardse R, Lodder BN, et al. Dendritic and Axonal Architecture of Individual Pyramidal Neurons across Layers of Adult Human Neocortex. Cereb Cortex. 2015;25: 4839-4853. doi:10.1093/cercor/bhv188 
85. Hashemi E, Ariza J, Rogers H, Noctor SC, Martínez-Cerdeño V. The Number of Parvalbumin-Expressing Interneurons Is Decreased in the Prefrontal Cortex in Autism. Cereb Cortex N Y N 1991. 2017;27: 1931-1943. doi:10.1093/cercor/bhw021

86. Krienen FM, Goldman M, Zhang Q, C. H. del Rosario R, Florio M, Machold R, et al. Innovations present in the primate interneuron repertoire. Nature. 2020;586: 262-269. doi:10.1038/s41586-020-2781-z

87. 2010 Allen Institute for Brain Science. Allen Human Brain Atlas. Available from: human.brain-map.org. $\quad$ [cited $19 \quad$ Apr 2021]. Available: https://www.google.com/search?q=2010+Allen+Institute+for+Brain+Science.+Allen+Hum an+Brain+Atlas.+Available+from $\% 3 \mathrm{~A}+$ human.brain-

map.org.\&oq=2010+Allen+Institute+for+Brain+Science.+Allen+Human+Brain+Atlas.+Av ailable+from\%3A+human.brainmap.org.\&aqs=chrome..69i57.422j0j4\&sourceid $=$ chrome\&ie $=U T F-8$

88. Hodge RD, Bakken TE, Miller JA, Smith KA, Barkan ER, Graybuck LT, et al. Conserved cell types with divergent features in human versus mouse cortex. Nature. 2019;573: 61-68. doi:10.1038/s41586-019-1506-7

89. Carnevale T, Hines M, Hines M, Hines M. The NEURON Book. Cambridge University Press; 2006.

90. Destexhe A, Rudolph M, Fellous JM, Sejnowski TJ. Fluctuating synaptic conductances recreate in vivo-like activity in neocortical neurons. Neuroscience. 2001;107: 13-24. doi:10.1016/s0306-4522(01)00344-X

91. Teleńczuk B, Dehghani N, Le Van Quyen M, Cash SS, Halgren E, Hatsopoulos NG, et al. Local field potentials primarily reflect inhibitory neuron activity in human and monkey cortex. Sci Rep. 2017;7: 40211. doi:10.1038/srep40211

92. Yu J, Hu H, Agmon A, Svoboda K. Recruitment of GABAergic Interneurons in the Barrel Cortex during Active Tactile Behavior. Neuron. 2019;104: 412-427.e4. doi:10.1016/j.neuron.2019.07.027

93. McCann H, Pisano G, Beltrachini L. Variation in Reported Human Head Tissue Electrical Conductivity Values. Brain Topogr. 2019;32: 825-858. doi:10.1007/s10548-019-00710-2

94. Welch P. The use of fast Fourier transform for the estimation of power spectra: A method based on time averaging over short, modified periodograms. IEEE Trans Audio Electroacoustics. 1967;15: 70-73. doi:10.1109/TAU.1967.1161901

95. Virtanen P, Gommers R, Oliphant TE, Haberland M, Reddy T, Cournapeau D, et al. SciPy 1.0: fundamental algorithms for scientific computing in Python. Nat Methods. 2020;17: 261272. doi:10.1038/s41592-019-0686-2

96. Harris CR, Millman KJ, van der Walt SJ, Gommers R, Virtanen P, Cournapeau D, et al. Array programming with NumPy. Nature. 2020;585: 357-362. doi:10.1038/s41586-020-2649-2 
97. Gramfort A, Luessi M, Larson E, Engemann DA, Strohmeier D, Brodbeck C, et al. MEG and EEG data analysis with MNE-Python. Front Neurosci. 2013;7. doi:10.3389/fnins.2013.00267

98. McBride D, Barrett SP, Kelly JT, Aw A, Dagher A. Effects of expectancy and abstinence on the neural response to smoking cues in cigarette smokers: an fMRI study. Neuropsychopharmacol Off Publ Am Coll Neuropsychopharmacol. 2006;31: 2728-2738. doi:10.1038/sj.npp.1301075

99. Koenigs M, Grafman J. The functional neuroanatomy of depression: Distinct roles for ventromedial and dorsolateral prefrontal cortex. Behav Brain Res. 2009;201: 239-243. doi:10.1016/j.bbr.2009.03.004 